\begin{tabular}{|c|l|}
\hline Title & $\begin{array}{l}\text { Structure determination of a novel protein by sul phur SA D using chromium radiation in combination with a new crystal } \\
\text { mounting method }\end{array}$ \\
\hline Author(s) & Kitago, Y u; W atanabe, Nobuhisa; Tanaka, Isao \\
\hline Citation & $\begin{array}{l}\text { Acta Crystall lographica Section D, 61(8), 1013-1021 } \\
\text { https://doi.org/L0.1107/S0907444905012734 }\end{array}$ \\
\hline Issue Date & 2005-08 \\
\hline Doc URL & http://hdl.handle.net/2115/8532 \\
\hline Rights & Copyright $\odot$ International Union of Crystallography \\
\hline Type & article (author version) \\
\hline File Information & LooplessMount.pdf \\
\hline
\end{tabular}

Instructions for use 


\title{
Structure determination of a novel protein by sulphur SAD using chromium radiation in combination with a new crystal mounting method
}

\author{
Yu Kitago, Nobuhisa Watanabe ${ }^{*}$ and Isao Tanaka \\ Division of Biological Sciences, Graduate School of Science, Hokkaido University, \\ Japan.E-mail: nobuhisa@sci.hokudai.ac.jp
}

Synopsis A novel protein structure was solved by S-SAD using in-house chromium radiation. To reduce X-ray absorption of the cryo-buffer and cryoloop, a novel free crystal mounting technique was developed.

Abstract A novel and easy crystal mounting technique was developed for the sulphur SAD

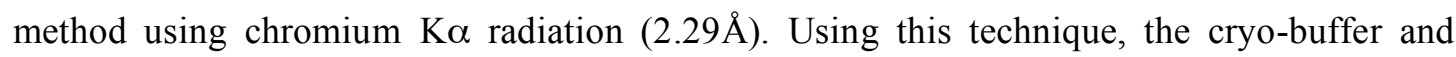
cryoloop around the protein crystal can be removed before data collection to eliminate their X-ray absorption. The superiority and the reproducibility of the datasets with our mounting technique were demonstrated using tetragonal hen egg-white lysozyme crystals. The structure of a novel protein, PH1109, from Pyrococcus horikoshii OT3 was solved using this technique. At the wavelength of chromium K $\alpha$ radiation, anomalous signals, $<|\Delta \mathrm{F}|>|<| \mathrm{F} \mid>$ of PH1 109 is expected to be $1.72 \%$, as this protein of 144 residues includes 4 methionines and 2 cysteines. Sulphur SAD phasing was performed using SHELXD and SHELXE. In the case of the dataset obtained using our novel crystal mounting technique, $54.9 \%$ of all residues were built with side-chains automatically by RESOLVE. On the other hand, only $16.0 \%$ were built with side-chains for the dataset collected using the standard cryoloop. These results indicated that our crystal mounting technique was superior to the standard loop mounting method for measurement of small anomalous differences at longer wavelength, and yielded better results in sulphur substructure solution and initial phasing. The present study demonstrated that the sulphur SAD method with a chromium source becomes enhanced more practical for macromolecular structure determination using our crystal mounting technique.

Keywords: sulphur; single-wavelength anomalous scattering; chromium radiation; crystal mounting method.

\section{Introduction}

Use of the single wavelength anomalous diffraction method (the SAD method, sometimes called the SAS method) is becoming increasingly common in protein crystallography. Many 
protein structures have been solved by the SAD method with heavy atoms such as $\mathrm{Se}, \mathrm{Pt}, \mathrm{Au}$, $\mathrm{Hg}$ etc. using synchrotron X-rays near their absorption edge. The use of sulphur atoms for SAD phasing is especially attractive because sulphur atoms are present in almost all proteins (as methionine or cysteine residues), and thus neither modification, such as Se-Met substitution, nor heavy atom soaking is necessary for structure analysis. However, the long wavelength of the sulphur K-absorption edge (5.02 $\AA$ ) hampers data collection at wavelengths close to the absorption edge of sulphur, and the small anomalous effects at usual wavelength ranges limit the use of sulphur atoms for SAD phasing. Thus, although sulphur SAD using $\mathrm{Cu}$ K $\alpha$ was used successfully to solve the crambin structure (Hendrickson \& Teeter, 1981), there have been few reports of subsequent applications of this method. However, over the last five years there have been several reports of successful application of sulphur SAD phasing. These experiments were carried out using $\mathrm{Cu} \mathrm{K} \alpha$ radiation (1.54 $\AA$ ) (Bond et al., 2001; de Graaff, et al., 2001; Yang et al., 2001; Lemke et al., 2002; Debreczeni et al., 2003), or synchrotron radiation at a wavelength of $1.54 \AA$ to $1.77 \AA$ (Dauter et al., 1999; Liu et al., 2000; Gordon et al., 2001; Brown et al., 2002; Li et al., 2002; Micossi et al., 2002; Ramagopal et al., 2003; Lartigue et al., 2004; Sekar et al., 2004).

More recently, successful application of the sulphur SAD technique was reported using a X-rays generated at much longer wavelength (2.29 $\AA$ ) from a chromium target as compared to a standard $\mathrm{Cu}$ target rotating anode source $(1.54 \AA)$, (Chen et al., 2004) with an X-ray apparatus optimised for protein crystallography (Yang et al., 2003). As suggested by Wang (1985), the longer wavelength from a chromium target is advantageous for sulphur SAD phasing because the $\Delta f^{\prime \prime}$ value of the sulphur atom is larger $\left(1.14 \mathrm{e}^{-}\right)$than that obtained with $\mathrm{Cu}$ radiation $\left(0.56 \mathrm{e}^{-}\right)$. However, the absorption coefficients of materials also become larger at longer wavelengths. Especially in standard protein crystallography where the crystal is mounted in a cryoloop with cryo-buffer, X-ray absorption by these materials sometimes prevents the detection of tiny anomalous signals with a high degree of accuracy. Therefore, the use of the longer wavelength is still controversial.

Here, we describe a novel technique for mounting crystals that eliminates absorption effects due to the cryo-buffer and cryoloop. This technique is especially useful for collecting data using longer wavelength X-rays for sulphur SAD phasing, and can be used easily as a conventional cryoloop. The usefulness of the new crystal mounting technique was demonstrated by tetragonal hen egg-white lysozyme crystals and by determining the structure of an unknown protein by using the anomalous signal alone from sulphur with Chromium $\mathrm{K} \alpha$ X-rays. 


\section{Methods}

\subsection{Sample preparation, diffraction data collection and processing}

Hen egg-white lysozyme (Seikagaku Kogyo, Tokyo) was purchased and used without further purification. The tetragonal crystals were obtained with the hanging drop vapour diffusion method at $20^{\circ} \mathrm{C}$. The protein $(60 \mathrm{mg} / \mathrm{mL})$ and reservoir volume ratio in the crystallization drop was 3:3 $\mu \mathrm{L}$, and the reservoir volume was $1 \mathrm{~mL}$ including $1.5 \mathrm{M} \mathrm{NaCl}$, $100 \mathrm{mM}$ sodium acetate buffer (pH4.2). Prior to flash-cooling, the crystals were rinsed in a cryo-protectant solution containing $30 \%(\mathrm{v} / \mathrm{v})$ glycerol for about 30 seconds. In order to demonstrate the superiority and reproducibility of our mounting method, several crystals of almost the same dimensions, about $0.2 \times 0.2 \times 0.15 \mathrm{~mm}$, were selected and used.

A novel protein PH1109 from Pyrococcus horikoshii OT3, used in this experiment was expressed in E. Coli, purified with HiTrap Q XL 5mL and HiLoad 26/60 superdex 200 pg (Amersham Biosciences), and concentrated to $10 \mathrm{mg} / \mathrm{mL}$ in a $10 \mathrm{mM}$ Tris- $\mathrm{HCl}$ buffer $\mathrm{pH} 8.0$ and $20 \mathrm{mM} \mathrm{NaCl}$. PH1 109 crystals were obtained with sitting drop vapour diffusion method under condition No. 37 of the Cryo I screening kit from deCode Genetics (Reykjavik, Iceland) consisting of $40 \%(\mathrm{v} / \mathrm{v})$ PEG-300, $0.1 \mathrm{M}$ cacodylate buffer $(\mathrm{pH} 6.5)$ and $0.2 \mathrm{M}$ calcium acetate. The protein and reservoir volume ratio in the crystallisation drop was 1:1 $\mu \mathrm{L}$, and the reservoir volume was $100 \mu \mathrm{L}$. The crystals were grown to a size of $0.1 \times 0.1 \times 0.3 \mathrm{~mm}$ in the sitting drop at $20^{\circ} \mathrm{C}$. These crystals belonged to the space group $P 6_{5} 22$ and had unit cell dimensions of $a=b=70.0 \AA, c=144.2 \AA$. The asymmetric unit contains one protein molecule and estimated solvent content is $58 \%$. The overall $\langle|\Delta \mathrm{F}|>|<| \mathrm{F}|>$ calculated from the amino acid sequence was $1.72 \%$ when the only sulphur atoms except the 1 st Met were taken into account as anomalous scatterers. For data collection, crystals were mounted either using the novel device described in the next section or by standard cryoloop. The diffraction data to a resolution of $2.17 \AA$ were collected using an in-house X-ray source; Rigaku FR-E SuperBright with a $\mathrm{Cu} / \mathrm{Cr}$ dual target (40 kV, $40 \mathrm{~mA}$ for $\mathrm{Cr}$ ), Osmic Confocal MaxFlux optics optimised for chromium (Cr CMF, Yang et al., 2003) and a Rigaku R-AXIS VII imaging plate detector. A $0.5 \mathrm{~mm}$ collimator was used to keep the whole crystal was always bathed in the X-ray beam. The black paper on the front of R-AXIS VII was replaced by a carbon-filled thin polymer film to decrease absorption. A post-sample helium path (Yang et al., 2003) and a collimator extension cap were also used to reduce absorption and scattering by the air. The data collection protocol, including highly redundant measurement, was the standard one for single-wavelength experiments. A total of 360 images with $1.0^{\circ}$ oscillation were collected for lysozyme, and a total of 720 images with $0.5^{\circ}$ oscillation were collected for PH1109 with a crystal-to-detector distance of $80 \mathrm{~mm}$. An exposure time for PH1 109 was 1 minute, and that for lysozyme crystals was summarized in Table 1. For comparison, datasets were also 
collected with the same apparatus but using a standard cryoloop. The collected intensities were indexed, integrated, absorption corrected, scaled and merged using HKL2000 (Otwinowski \& Minor, 1997) with "scale anomalous" flag to keep Bijvoet pairs separate.

\subsection{Loopless and bufferless mount for eliminating X-ray absorption}

As described above, the X-ray absorption coefficients of materials increase at longer wavelengths. Therefore, diffraction intensities measured at low temperatures may be influenced strongly by the frozen buffer solution and the cryoloop around the crystal. To eliminate the X-ray absorption by the cryo-buffer and cryoloop, we have developed a novel technique for mounting a protein crystal without them. For this purpose, a new tool was devised based on the free mounting technique of protein crystals reported by Kiefersauer et al. $(1996 ; 2000)$, which was originally used to improve the resolution limit of diffraction by accurately controlled humidity changes. As in their method, a glass micropipette was made using a Narishige PC-10 pipette puller (Narishige Scientific Instrument Laboratory, Japan), and the tip of the micropipette was cut and ground using a Narishige MF-900 microforge and an EG-400 micropipette grinder. Our technique is novel in that the a nylon loop is glued directly onto the tip of the micropipette and fixed as if the micropipette tip is located in the loop, so the solution caught in the loop can be aspirated through the micropipette just before flash freezing (Fig. 1(a)). A mounting base for holding the micropipette airtight was also made (Fig. 1(b)). Protein crystals can be picked up from the crystallisation drop using the loop at the tip of the pipette, and the cryo-buffer in the loop can be removed by aspiration before flash freezing the crystal on a goniometer head of the diffractometer. When the cryobuffer is removed by aspiration, the protein crystal is usually located on the tip of the micropipette as shown in Fig. 1(c). The remaining cryoloop can be torn off with a small hook or forceps with careful attention paid to the crystal.

The actual procedure for mounting a crystal was as follows. Before the crystal was picked up, the micropipette with the loop was fixed on the mounting base, and a soft tube for aspiration was connected to the base. A crystal in the cryo-buffer was picked up using the loop at the tip of the micropipette, and the device was mounted on the goniometer head intercepting the cryo-stream as in the standard procedure. The cryo-buffer in the loop was removed immediately by aspiration, and the crystal was flash frozen on the tip of the micropipette with a stream of cold nitrogen. Finally, the glued cryoloop was removed using a micro-hook made of metal wire without disturbing the cold stream. In this way, we succeeded in freezing and holding the crystal without buffer or loop and avoiding dehydration. In the most cases, this crystal mounting procedures are not difficult and rate of failure would be almost same as the standard cryoloop mounting technique. 


\subsection{SAD Phasing of PH1109 protein}

To estimate the accuracy of measurement of anomalous differences, the experimental values of $\langle|\Delta \mathrm{F}|>|<| \mathrm{F}|>$ and $\langle|\Delta \mathrm{F}|>|<\sigma(\Delta \mathrm{F})>$ were plotted against the resolution bin. The values of $\langle|\Delta \mathrm{F}|>|<| \mathrm{F}|>$ were compared with the theoretical value calculated from the elemental composition estimated from the amino acid sequence (Dauter et al., 1999; 2002). The positions of anomalous scatterers were located using SHELXD (Sheldrick et al., 2001) after analysing the substructure structure factors by SHELXC (Sheldrick, 2003). The initial phases were estimated by SHELXE (Sheldrick, 2002) with the sites, and improved by density modification with a solvent content of 0.58. After SHELXE, the initial model was built automatically by RESOLVE (Terwilliger, 2000; 2003) with the build_only option. The initial model was extended and refined automatically by Lafire (Yao et al., in preparation), which implements CNS programs (Brünger et al., 1998) for the refinement. The model and the improved electron density maps were inspected using XtalView (McRee, 1992).

\section{Results and discussion}

\subsection{Superiority of the mounting technique}

In order to demonstrate the superiority of our mounting technique, six datasets were collected with tetragonal hen egg-white lysozyme crystals, of which two were collected with our mounting technique (named NoLoop1 and 2) and four datasets were collected using standard cryoloop (Loop1 - 4). Two of the four datasets with a cryoloop (Loop3 and 4) were collected using about 1.5 times larger crystals than other four crystals. The collected intensities were processed by HKL2000 and diffraction data statistics are shown in Table 1. The total number of measured reflections and completeness are almost the same for nine datasets. Statistics of each dataset, such as $\mathrm{R}_{\text {sym }}, \mathrm{I} / \sigma \mathrm{I},\langle|\Delta \mathrm{F}|>|<| \mathrm{F}|>$ and $\langle|\Delta \mathrm{F}|>|<\sigma(\Delta \mathrm{F})>$ were plotted against resolution bins and shown in Figure 2. The correration coefficients (CC/all) of SHELXD were also calculated against resolution bins as reported by Fu et al. (2004). All plots show that the data with our mounting technique gave better results. The behaviour of the plots of Bijvoet ratio $<|\Delta \mathrm{F}|>|<| \mathrm{F} \mid>$ were not so clear, but tendency of overestimate of anomalous differences at higher resolution can be seen for the datasets with cryoloop. Intriguingly, $I / \sigma I$ plots were not improved, even when larger size of crystals were selected.

\subsection{Structure solution of $\mathrm{PH} 1109$ protein}

Data collection statistics were summarized in Table 2. Various statistics against resolution bin were shown in Figure 3. As same as the lysozyme test case, the experimental plots of Bijvoet ratio, $\langle|\Delta \mathrm{F}|>|<| \mathrm{F}|>$, showed a smooth behaviour, which coincided well with the 
theoretical curve calculated from the amino acid sequence in the resolution range of 20 $2.17 \AA$, and difference of the data quality between two datasets were not clearly observed. However, the difference between the two datasets can be seen as expected in the signal to noise ratio, I/ $/ \mathrm{I}$ and $\langle|\Delta \mathrm{F}|>|\langle\sigma(\Delta \mathrm{F})>$. The dataset collected using our technique gave better statitics as the results of lysozyme crystals.

Consequently, the two mounting methods showed clear differences in sulphur substructure determination and the phasing process. The correlation coefficients CC/all values from SHELXD showed significant superiority of the novel mounting technique. The higher CC/all values indicated that the dataset measured using our method had more accurate anomalous signals and was more useful to produce the anomalous scattering substructure than the standard cryoloop mounting procedure.

Although PH1 109 has 6 sulphurs (4 Met and 2 Cys, except for the 1st Met), SHELXD gave 11 sites as anomalous scatterers. Comparison with the final structure indicated that 2 of these 11 sites were phosphorus of coenzyme A, which binds to the PH1109 molecule. The $\Delta f^{\prime}$ value of a phosphor atom is $0.899 \mathrm{e}^{-}$at a wavelength of $2.29 \AA$, and was sufficient to be found by SHELXD. The other 3 sites seemed to be a kind of ions because these were located on the surface of the protein molecule. One site may be calcium cations because calcium acetate was included in the crystallisation condition and this site was coordinated by seven ligands. The other 2 of 3 ions may have been chloride anions which were included in the sample buffer. The remaining 6 sites were sulphur atoms of the PH1 109 molecule. After SAD phasing and density modification by SHELXE, the electron density map obtained was of quite good quality as shown in Figure 4(a). Most of the secondary structures and some distinctive side chains, such as Tyr or Trp, were clearly identified. As a result of auto building by RESOLVE, 109 of the 144 residues were traced, and of these 79 residues were identified with their side chains (Fig. 5(a)). The final model after automatic model extension and refinement by Lafire contained 142 of 144 residues (Fig. 5(b)). The C-terminal two residues are disordered and were not found in the electron density map. However, the bound coenzyme A was found in the electron density map.

The differences of the phasing results between the datasets using the novel mounting technique and that using the standard cryoloop are summarized in Table 3. The large differences in number of residues built by RESOLVE were clearly demonstrated that our crystal mounting method is superior to the conventional method in practical use. Almost all of the models were traced automatically using the data measured with our crystal mounting technique. On the other hand, the data using the standard cryoloop gave many short fragments, as shown in Figure 5(c). Compared with the electron density map calculated by the 
datasets of our mounting method, the map of the dataset using standard cryoloop is poorer as shown in Figure 3.

As shown in Table 2, completeness of the dataset collected using standard cryoloop was a little worse than the dataset with our mounting method, because the $\mathrm{c}^{*}$ axis was accidentally aligned to the spindle of the diffractometer. In order to remove this bias, only the same reflections as the standard cryoloop dataset were extracted from the dataset with our mounting method, and the same analysis was performed. The results and statistics are also summarized in Table 3. Although the result of RESOLVE auto-tracing was a little worse than that of the original dataset, the superiority of our crystal mounting method was kept as shown in Fig. 5 and Table 3.

The result of the present study suggested that absorption correction by SCALEPACK could not correct the measured intensity adequately when the standard cryoloop was used with longer wavelength X-ray, because the levels of X-ray absorption of the cryo-buffer and the nylon loop were much larger and their shape were not related to those of the protein crystal inside them. The scale factor estimated by SCALEPACK for the data obtained using the standard cryoloop and our device are plotted together in Figure 6. The dataset obtained using the standard cryoloop showed a large amplitude of modulation due to the frozen cryo-buffer and the cryoloop. On the other hand, the dataset obtained using our technique showed a smooth behaviour. These results clearly indicated that the effects of absorption by the cryobuffer and the cryoloop were decreased markedly using our mounting technique. Using our mounting technique, the absorption correction function could be estimated for the protein crystal itself and applied appropriately to the measured intensity data.

\section{Conclusions}

The results of the present study indicated that our crystal mounting technique can effectively increase the accuracy of measured anomalous differences. The results obtained using our technique showed clear superiority to those obtained with the standard cryoloop in structure determination by the sulphur SAD method. We succeeded in determining the structure of $\mathrm{PH} 109$ protein by the sulphur SAD method using an in-house chromium X-ray source and the novel crystal mounting technique. We have actually solved three other novel protein structures, two of which have a molecular weight of $68 \mathrm{kDa}$ and $84 \mathrm{kDa}$, using our mounting method (manuscripts in preparation). The estimated Bijvoet ratios, $\langle|\Delta \mathrm{F}|>|<| \mathrm{F}|>$, of each protein were $1.04 \%$ and $1.68 \%$, respectively. We think the sulphur SAD method using $\mathrm{Cr} \mathrm{K} \alpha$ radiation will become a practical means for determination of the structures of native proteins with our novel crystal mounting method. 


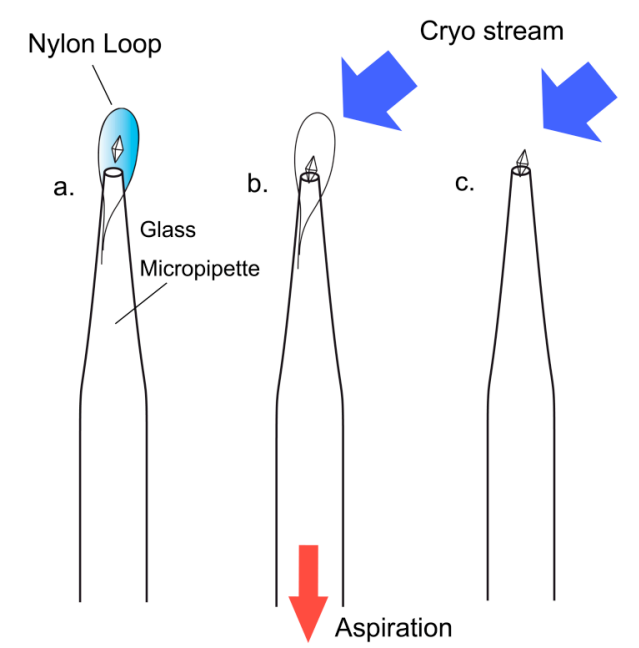

(a)
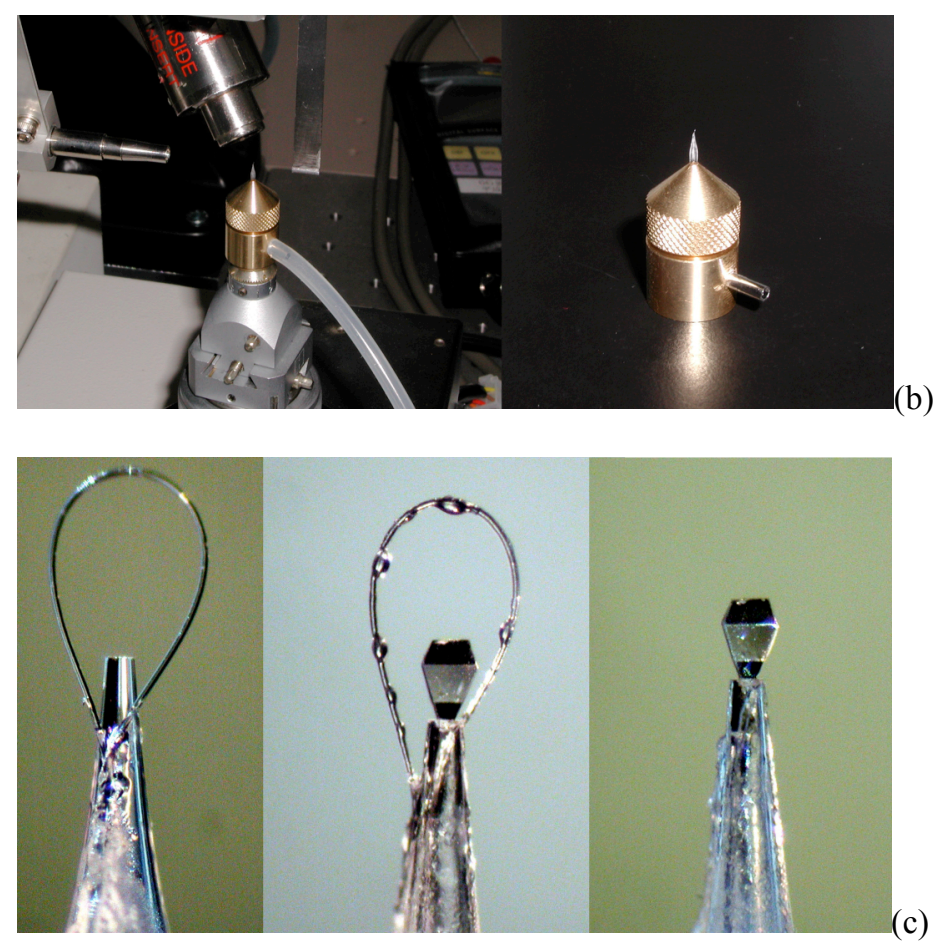

Figure 1 (a) A schematic illustration of the novel mounting technique. a. Picking up a protein crystal using nylon loop at the top of glass micropipette, and mounting the tools on the goniometer head intercepting the cryo-stream. b. Aspirating the cryo-buffer through the micropipette and flushing with the cold nitrogen stream. c. Removing the nylon-loop under cryo-conditions. (b) The mounting base for the novel mounting technique. This tool can hold the micropipette and aspirate the cryo-buffer through the flexible tube. (c) Left: The tip of the micropipette and nylon loop before picking up a protein crystal. The nylon loop was glued to the tip of the micropipette. Middle: The tip of the micropipette and a protein crystal after aspirating the cryo-buffer and freezing. Right: The tip of the micropipette and a protein crystal after removing the nylon loop. In the middle and right pictures, a thaumatin (SIGMA T7638) crystal was used as the sample. 
(a)

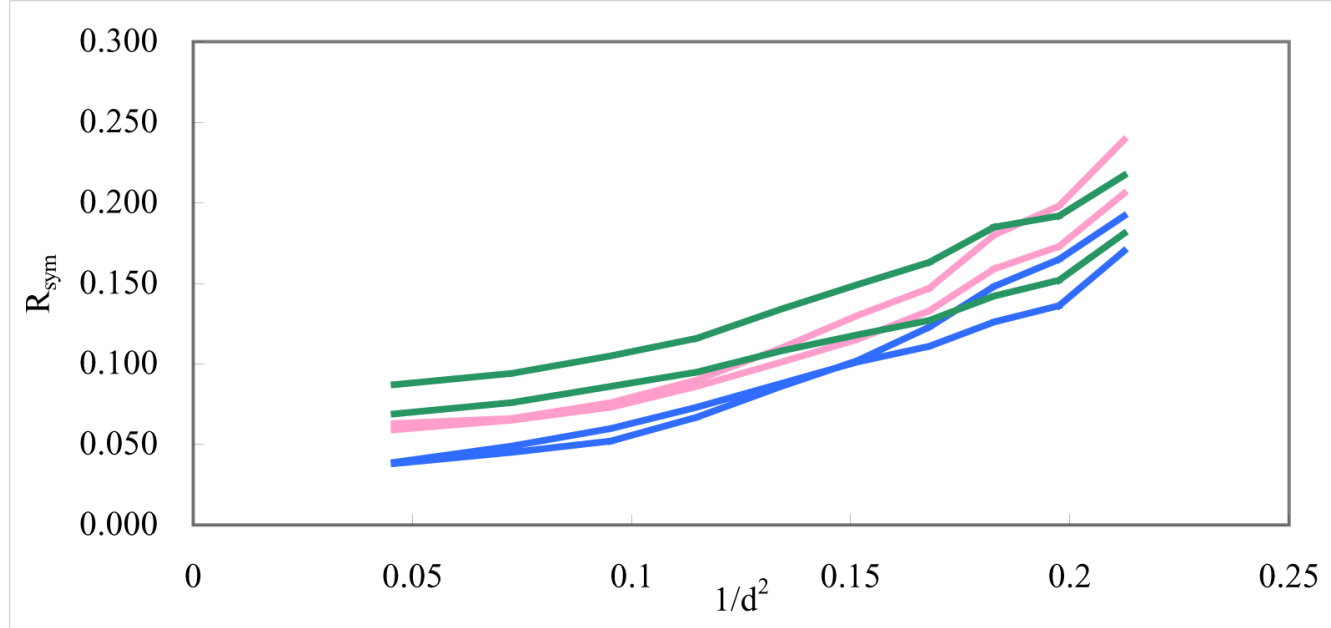

(b)

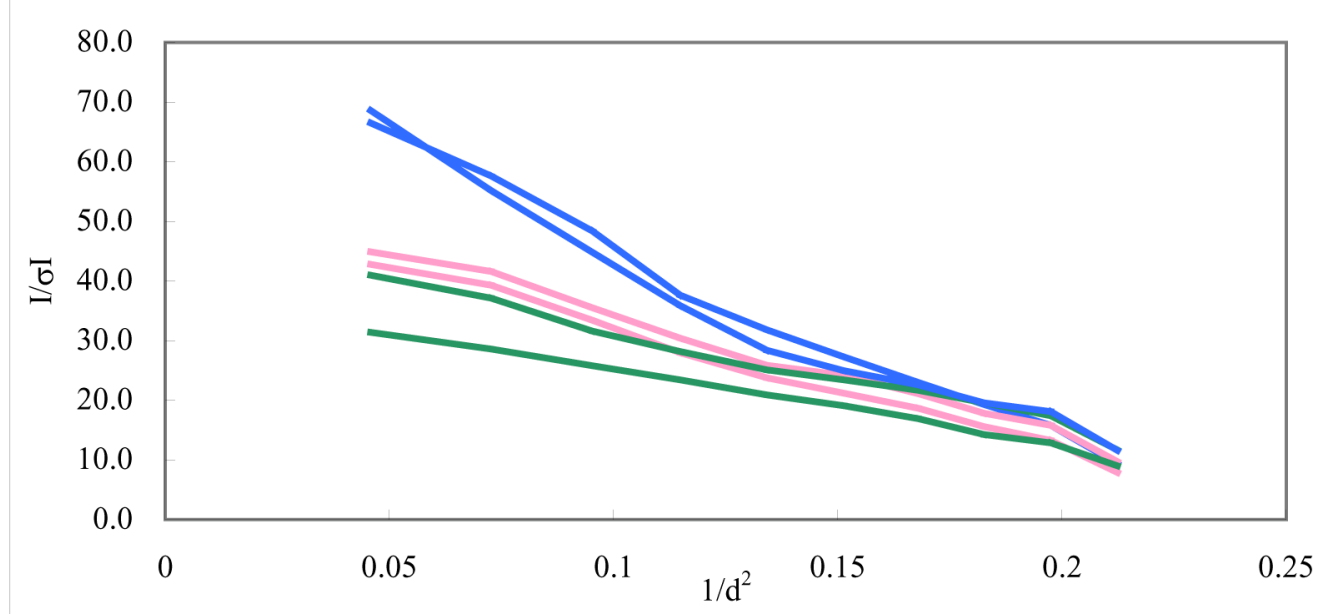

(c)

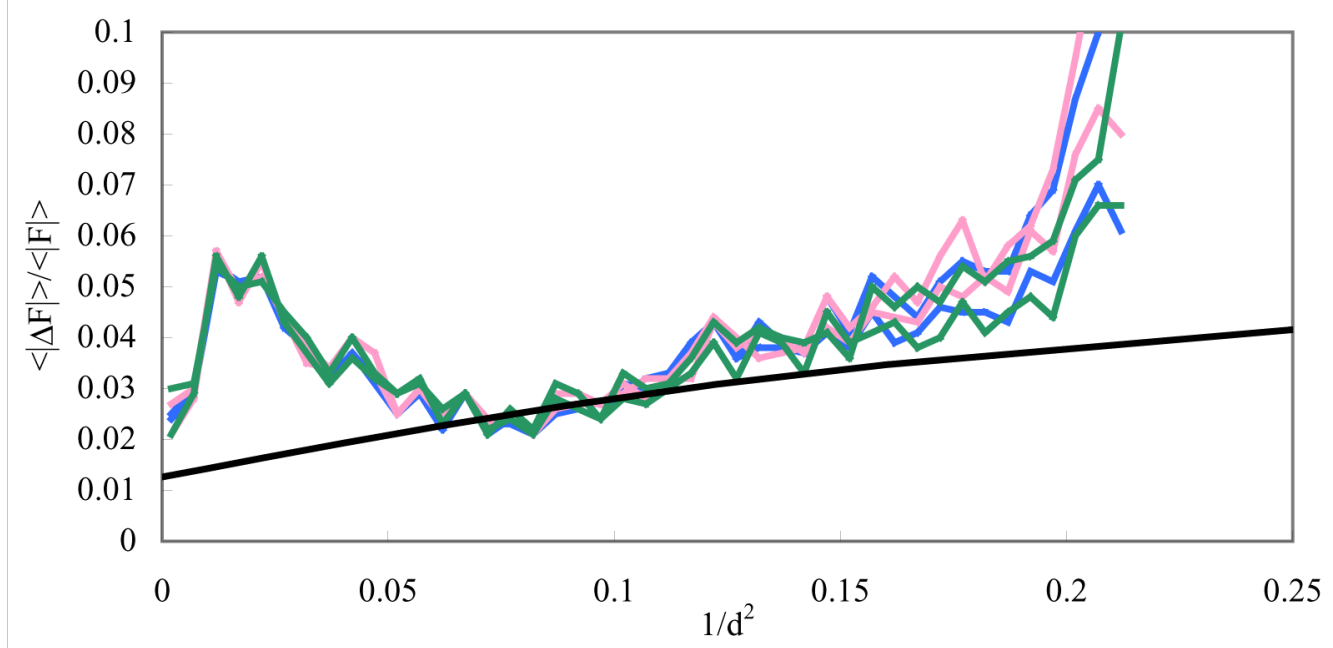

(d) 


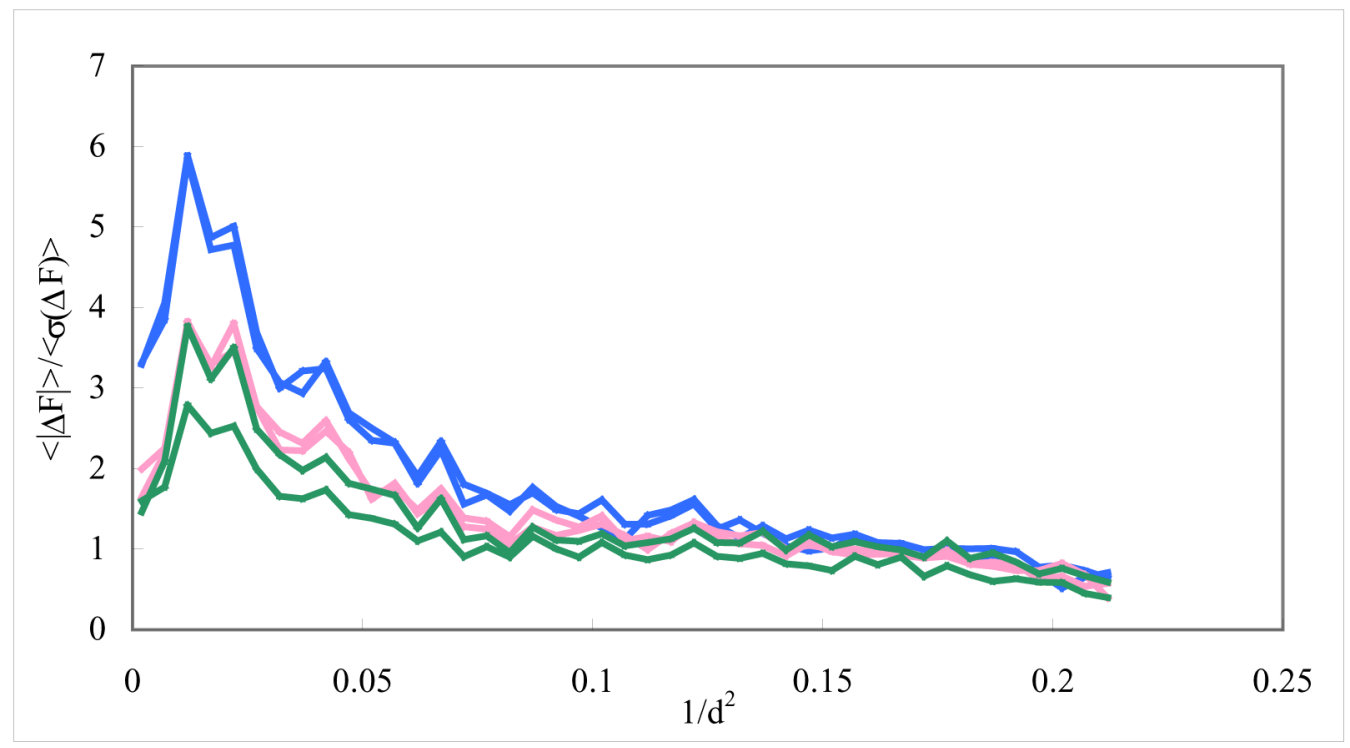

(e)

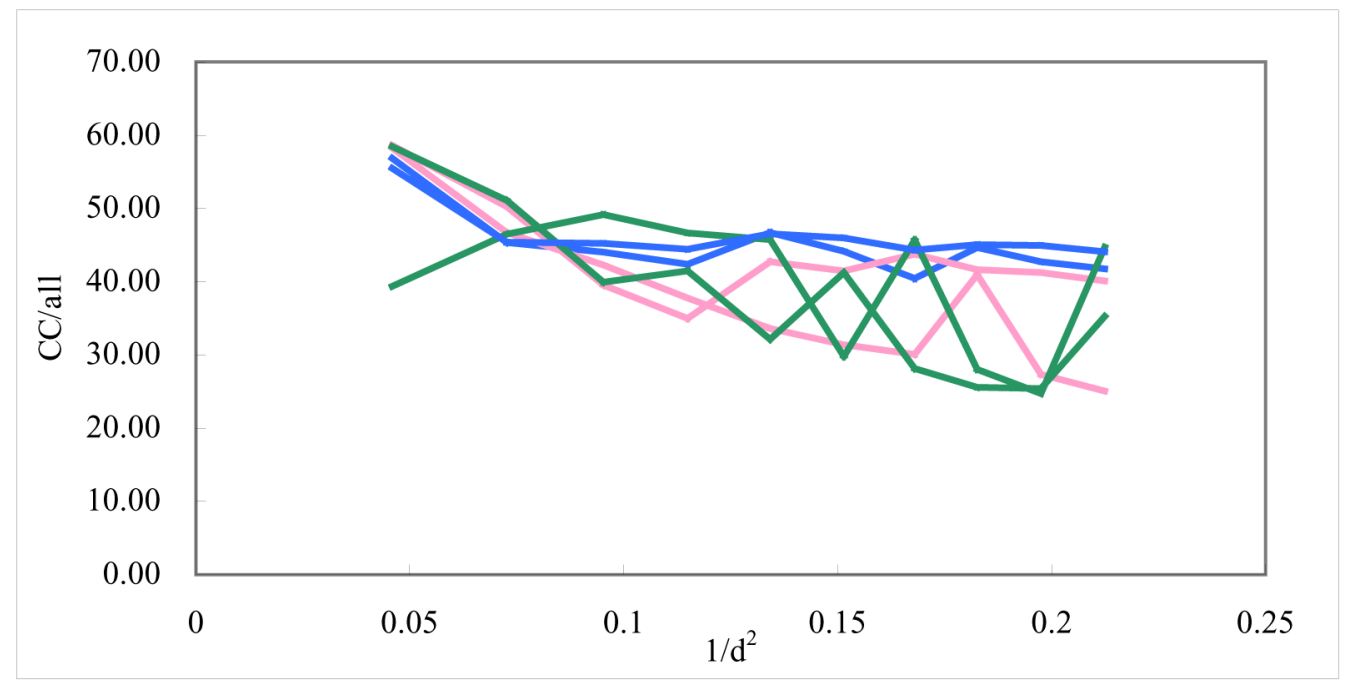

Figure 2 Statistics against resolution bin on tetragonal lysozyme. (a) $\mathrm{R}_{\text {sym }}$, (b) $\mathrm{I} / \sigma \mathrm{I}$, , (c) $<|\Delta \mathrm{F}|>|<| \mathrm{F} \mid>$, (d) $\langle|\Delta \mathrm{F}|>|\langle\sigma(\Delta \mathrm{F})>$, (e) CC/all, respectively. Blue: NoLoop 1 and 2. Pink: Loop 1 and 2. Green: Loop 3 and 4. Black in $\langle|\Delta \mathrm{F}|>|<| \mathrm{F}|>$ plot is theoretical value calculated from amino acid sequence. The values $\mathrm{CC} / \mathrm{all}$ were calculated with reflections up to the resolution shell plotted. $\mathrm{CC}$ is the correlation coefficient of the top solutions from SHELXD and this value is described in Fujinaga \& Read (1987) and Sheldrick et al. (2001) in detail. All other values were calculated with reflections in the listed resolution shell. 
(a)

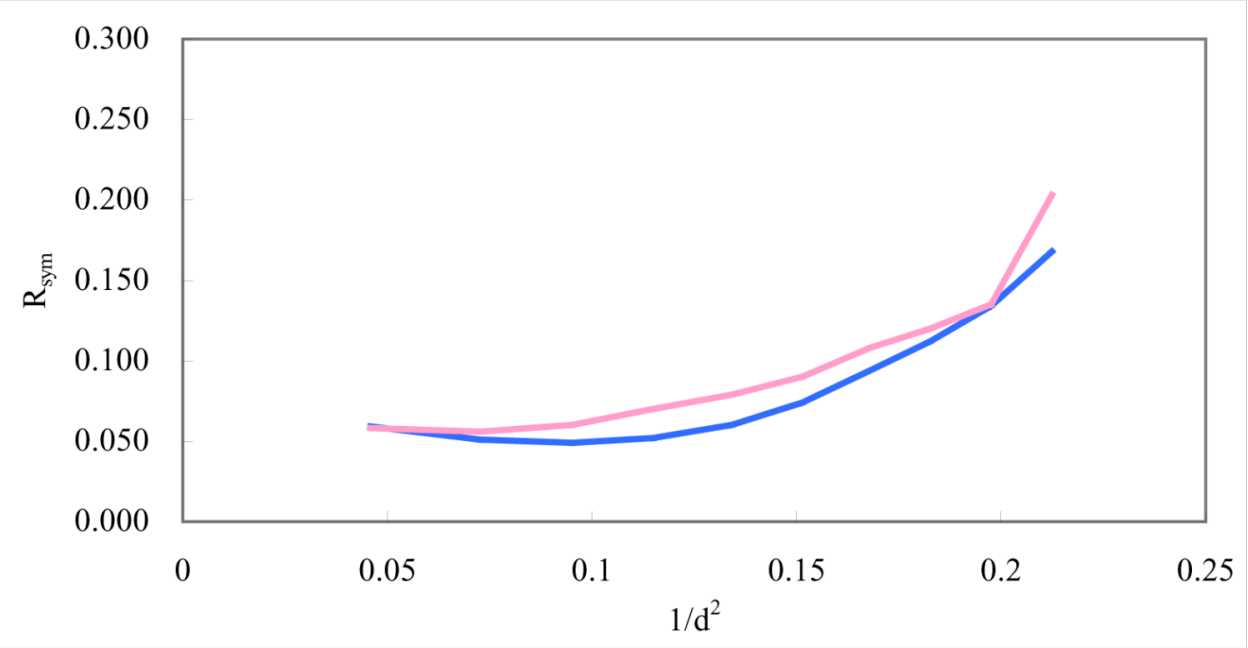

(b)

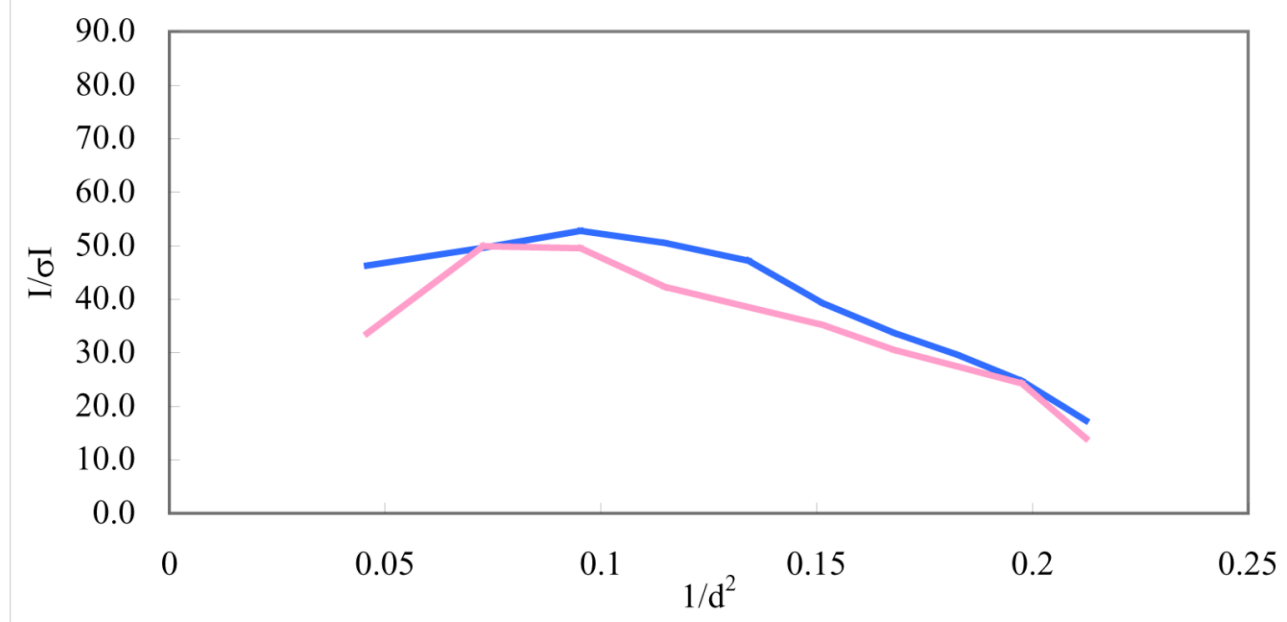

(c)

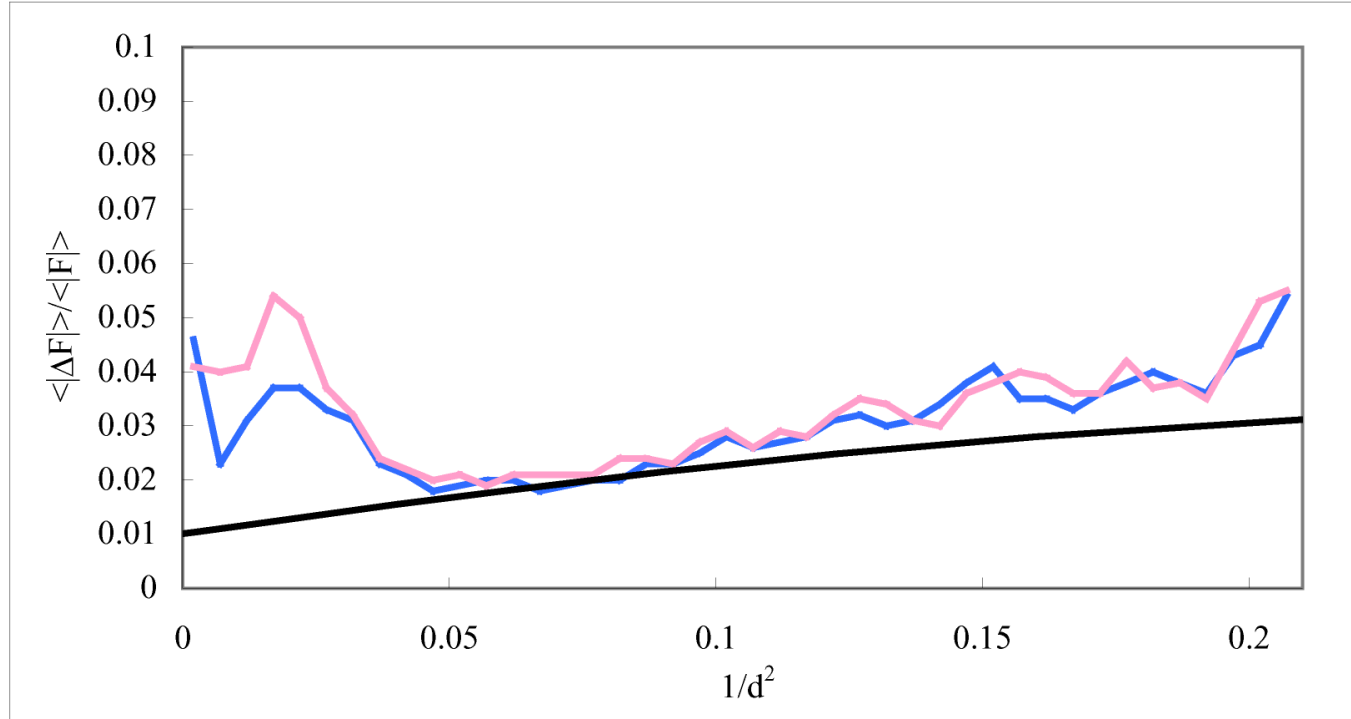


(d)

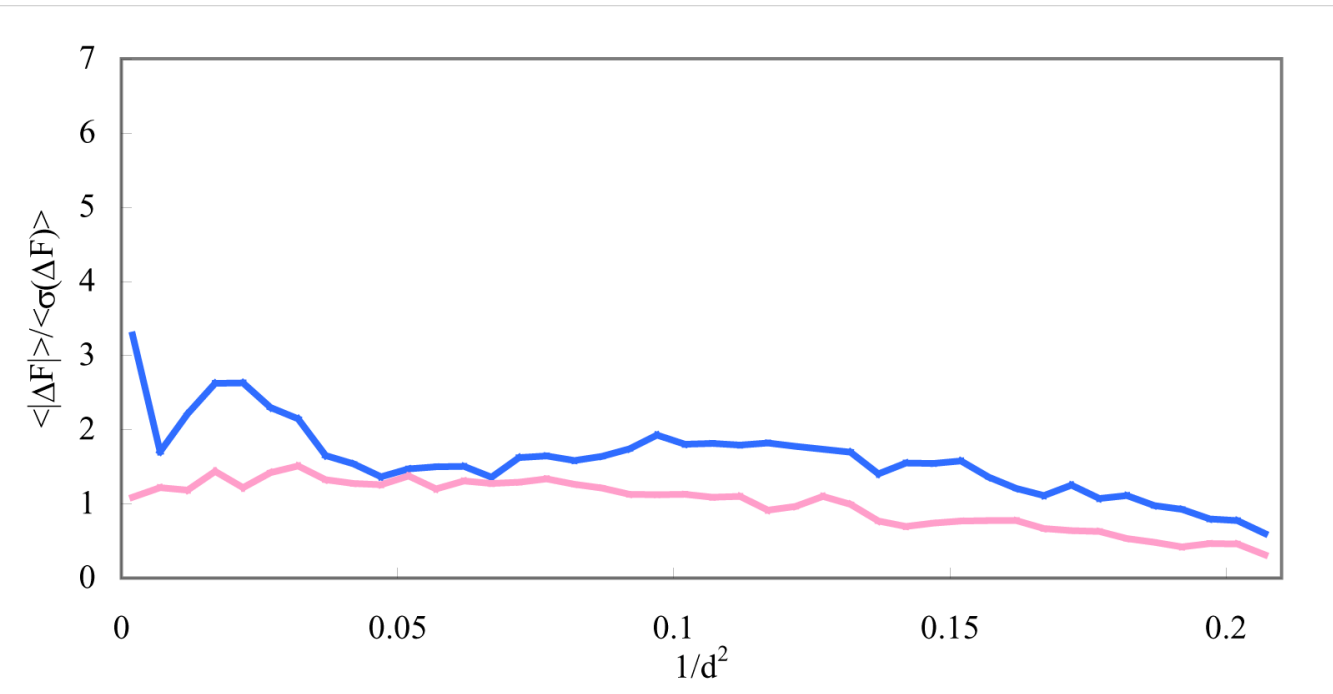

(e)

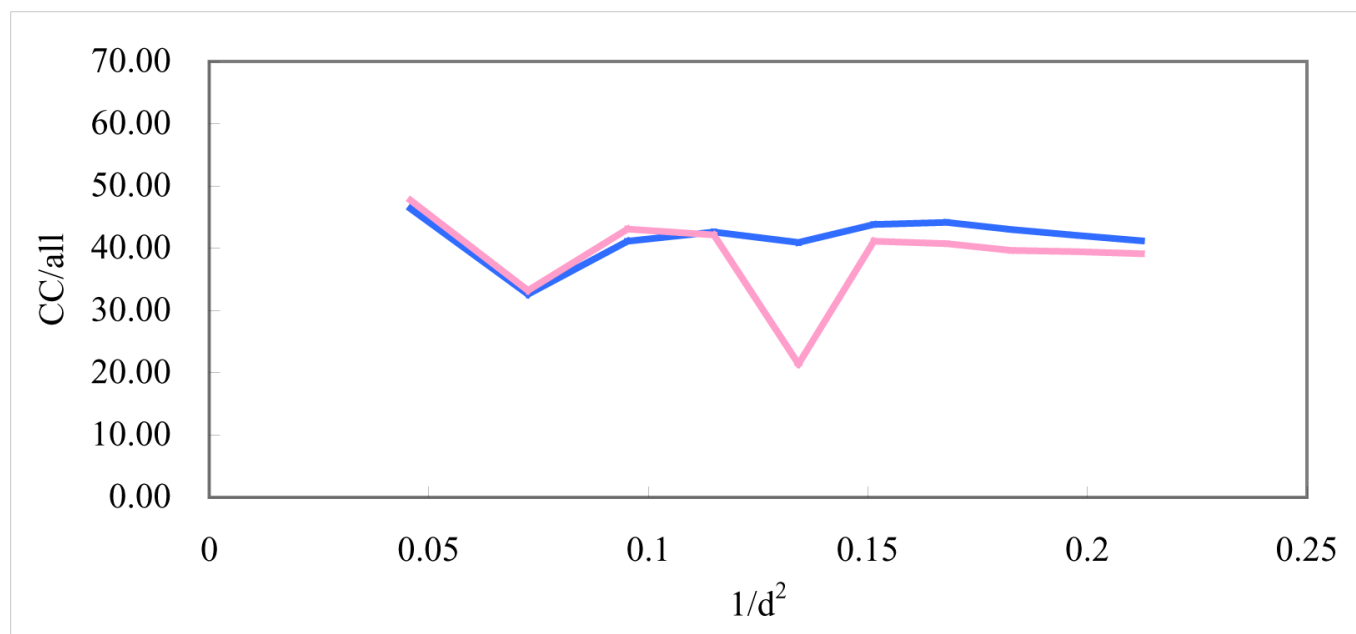

Figure 3 Dependence of the anomalous signal of PH1109 upon resolution. (a) $\mathrm{R}_{\text {sym }}$, (b) I/oI, (c) $\langle|\Delta \mathrm{F}|>|<| \mathrm{F}|>$, (d) $\langle|\Delta \mathrm{F}|>|<\sigma(\Delta \mathrm{F})>$ and (e) $\mathrm{CC} /$ all of two datasets obtained using the novel mounting technique and the standard cryoloop were plotted together against resolution bins. Blue: values calculated from the dataset obtained using the novel mounting technique. Pink: values calculated from the dataset obtained using the standard cryoloop. Black line in $\langle|\Delta \mathrm{F}|>|<| \mathrm{F}|>$ plot: theoretical $<|\Delta \mathrm{F}|>|<| \mathrm{F} \mid>$ values of PH1109 calculated from the amino acid sequence. The values CC/all were calculated as Figure 2. 


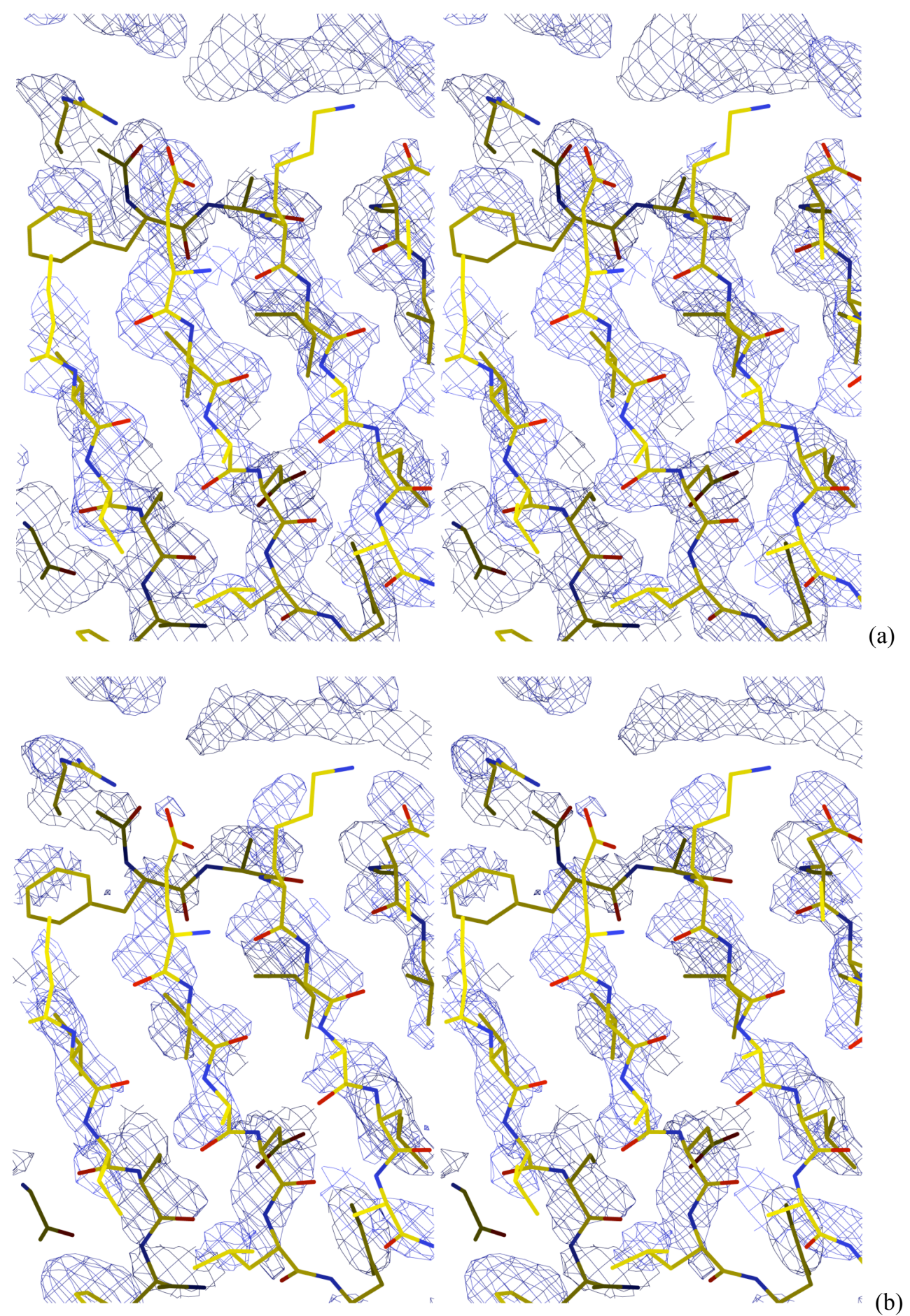

Figure 4 Stereo view of the initial electron density map of PH1 109 at the $2.0 \sigma$ level calculated using the phases estimated by SHELXE at a resolution of $2.17 \AA$. (a) Using our mounting method. (b) Using standard cryoloop method. The model in the figure is the refined model. 

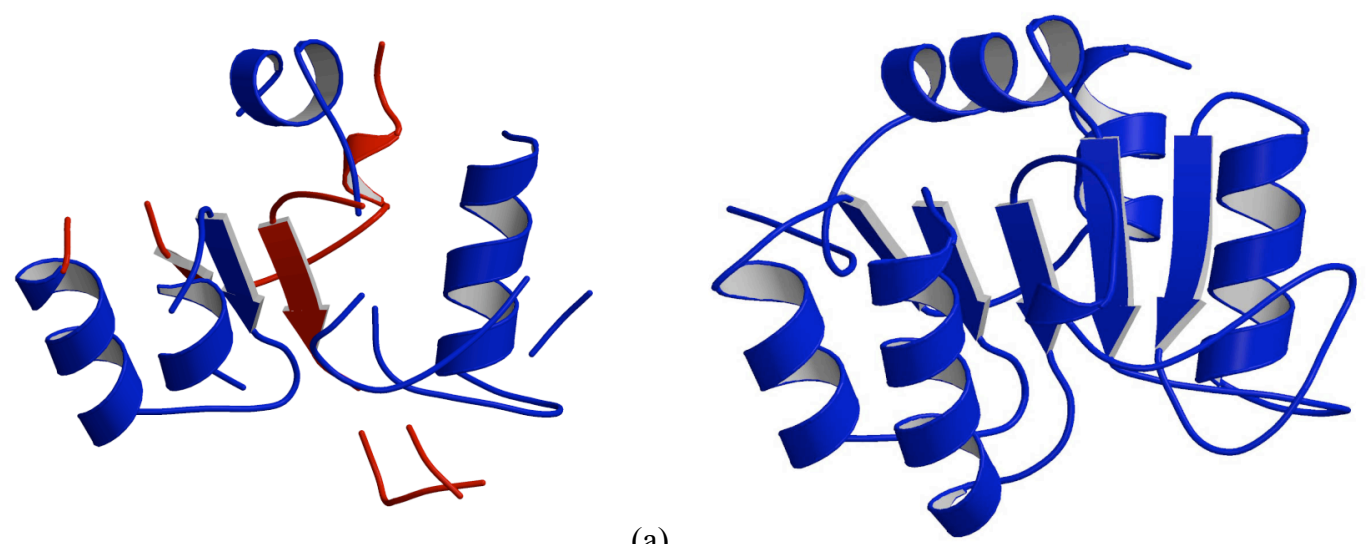

(a)

(b)
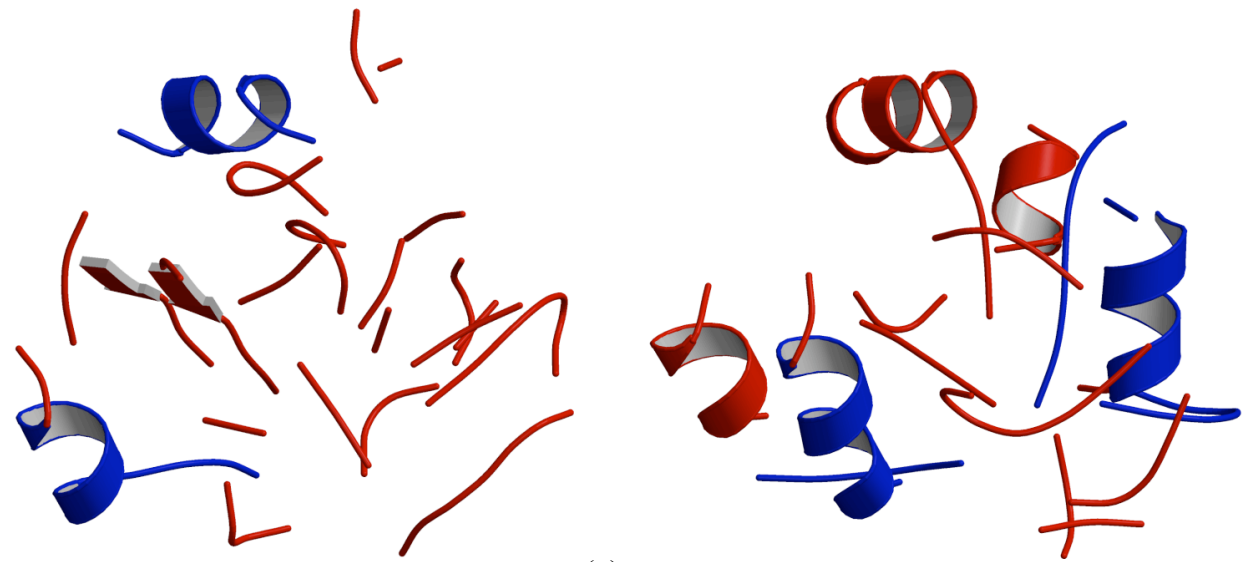

(c)

(d)

Figure 5 (a) Overview of the initial model of PH1109 built by RESOLVE using dataset with our crystal mounting method. The model identified without side chains is shown in red, and that with side chains is shown in blue. (b) The final model constructed by Lafire. (c) The initial model produced from the dataset obtained using the standard cryoloop built by RESOLVE. The colours are the same as in (a).

(d) The initial model produced using an extracted dataset that has only the same reflections as the dataset with the standard loop.

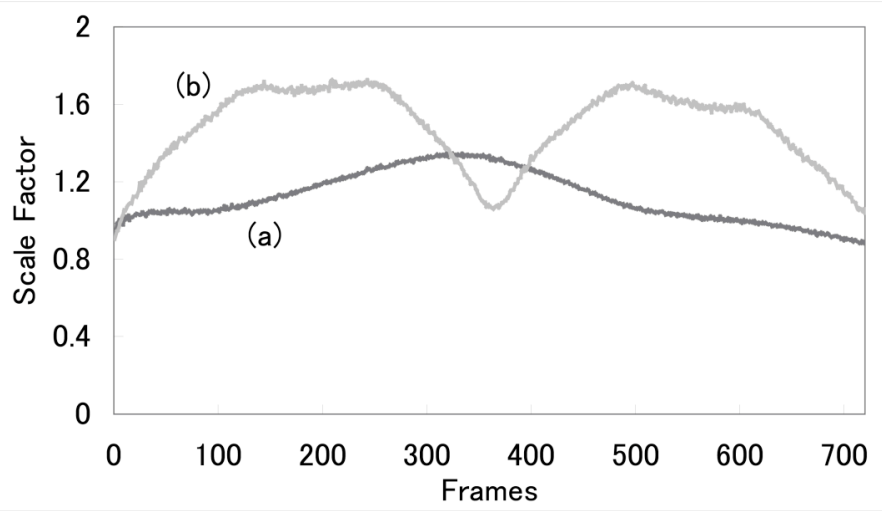

Figure 6 Scale factor plot of PH1 109 against frames. (a) The dataset obtained using the novel mounting technique. (b) The dataset obtained using the standard cryoloop. 
Table 1 Data collection statistics of tetragonal lysozyme.

\begin{tabular}{|c|c|c|c|c|c|c|}
\hline & NoLoop1 & NoLoop2 & Loop1 & Loop2 & Loop3 & Loop4 \\
\hline Crystal dimensions $\left[\mathrm{mm}^{3}\right]$ & $0.2 \times 0.2 \times 0.15$ & $0.2 \times 0.2 \times 0.15$ & $0.2 \times 0.2 \times 0.15$ & $0.2 \times 0.2 \times 0.15$ & $0.25 \times 0.25 \times 0.15$ & $0.25 \times 0.25 \times 0.15$ \\
\hline Crystal to detector distance $[\mathrm{mm}]$ & & & & 80.0 & & \\
\hline Oscillation range $\left[{ }^{\circ}\right]$ & & & & 1.0 & & \\
\hline Rotation range $\left[{ }^{\circ}\right]$ & & & & 360 & & \\
\hline Exp. time $[\mathrm{min}]$ & 0.5 & 1.0 & 1.0 & 1.0 & 1.0 & 1.0 \\
\hline Unit-cell parameters & $a=b=78.8, c=36.9$ & $a=b=78.8, c=36.9$ & $a=b=78.8, c=36.9$ & $a=b=78.8, c=36.9$ & $a=b=78.8, c=36.9$ & $a=b=78.8, c=36.9$ \\
\hline Space group & \multicolumn{6}{|c|}{$P 4_{3} 2_{1} 2$} \\
\hline Resolution limit & \multicolumn{6}{|c|}{$50.0-2.17(2.25-2.17)$} \\
\hline Reflections measured & 159480 & 165333 & 163221 & 160396 & 161119 & 165521 \\
\hline Unique reflections & 11692 & 11762 & 11796 & 11684 & 11775 & 11773 \\
\hline Completeness [\%] & $99.4(94.2)$ & $99.9(99.7)$ & $100.0(100.0)$ & $99.7(100.0)$ & $99.9(99.7)$ & $99.8(98.4)$ \\
\hline $\mathrm{R}_{\text {sym }}[\%]$ & $6.6(19.2)$ & $6.9(17.0)$ & 8.4 (20.6) & $8.9(23.9)$ & $11.4(21.7)$ & $9.3(18.1)$ \\
\hline Redundancy & $13.6(7.3)$ & $14.1(9.8)$ & $13.8(8.5)$ & $13.7(7.6)$ & $13.7(8.5)$ & $14.1(9.9)$ \\
\hline $\mathrm{I} / \sigma \mathrm{I}$ & $34.5(9.4)$ & $33.6(11.6)$ & $27.1(9.7)$ & 24.8 (7.9) & $20.4(9.0)$ & $26.0(11.7)$ \\
\hline Mosaicity & 0.486 & 0.394 & 0.566 & 0.526 & 0.403 & 0.420 \\
\hline
\end{tabular}

Table 2 Data collection statistics for PH1109.

\begin{tabular}{|c|c|c|}
\hline & Using the novel technique & Using the standard loop \\
\hline Crystal dimensions $\left[\mathrm{mm}^{3}\right]$ & $0.1 \times 0.1 \times 0.3$ & $0.1 \times 0.1 \times 0.3$ \\
\hline Crystal to detector distance [mm] & & 80.0 \\
\hline Oscillation range $\left[{ }^{\circ}\right]$ & & 0.5 \\
\hline Rotation range $\left[{ }^{\circ}\right]$ & & 360 \\
\hline Exp. time $[\mathrm{min}]$ & 1.0 & 1.0 \\
\hline Unit-cell parameters & $a=b=70.0, c=144.2$ & $a=b=69.8, c=142.9$ \\
\hline Space group & & $6_{5} 22$ \\
\hline Resolution limit & $50.0-2.17$ & $50.0-2.17$ \\
\hline Reflections measured & 439535 & 410744 \\
\hline Unique reflections & 20578 & 18901 \\
\hline Completeness [\%] & $98.3(94.5)$ & $91.5(79.1)$ \\
\hline $\mathrm{R}_{\text {sym }}[\%]$ & $5.8(16.8)$ & $6.7(20.3)$ \\
\hline Redundancy & $21.4(16.4)$ & $31.7(19.7)$ \\
\hline $\mathrm{I} / \sigma(\mathrm{I})$ & $39.6(17.3)$ & $33.2(14.0)$ \\
\hline Mosaicity & 0.687 & 0.470 \\
\hline
\end{tabular}

Table 3 The differences of several parameters between the datasets of PH1109. After density modification by SHELXE, the map CCs were compared between the datasets. The reference maps for map CC were calculated by the final models for each datasets without water molecules. (R of $27.0 \%$ 
and $\mathrm{R}_{\text {free }}$ of $23.0 \%$ for our mounting method, and 27.1 and $22.1 \%$ for standard cryoloop, respectively). The results for extracted data were also shown.

\begin{tabular}{lccc}
\hline & \multicolumn{2}{l}{ Using the novel technique } & Using the standard cryoloop \\
& Original & Extracted & \\
\hline $\begin{array}{l}\text { No. reflections used in SHELX } \\
\text { Total number of reflections }\end{array}$ & 20555 & 18954 & 18901 \\
Number of unique reflections & 11440 & 10459 & 10459 \\
\hline SHELXD & & & 38.72 \\
CC (all) & 41.21 & 41.49 & 18.64 \\
CC (weak) & 21.95 & 21.52 & 8.60 \\
PATFOM & 10.55 & 10.42 & 35.19 \\
\hline SHELXE & 38.19 & 38.99 & 23 \\
CC between $E_{\text {obs }}$ and $E_{\text {calc }}$ & & & 86 \\
\hline No. of Residues built by RESOLVE & 79 & 42 & 0.363 \\
with side chains & 33 & 69 & 0.593 \\
without side chains & & & \\
\hline Map CC with refined map & 0.515 & 0.497 & 0.808 \\
The map after phasing & 0.830 & & \\
The map after density modification & & & \\
\hline
\end{tabular}

Acknowledgements We thank Dr. Min Yao for allowing us to use PH1109 samples for this study. This work was supported by a research grant from the National Project on Protein Structural and Functional Analysis from the Ministry of Education, Culture, Sports, Science and Technology of Japan.

\section{References}

Bond, C. S., Shaw, M. P., Alphey, S. \& Hunter, W. N. (2001). Acta Cryst. D57, 755-758.

Brown, J., Esnouf, R. M., Jones, M. A., Linnell, J., Harlos, K., Hassan, A. B. \& Jones, E. Y. (2002). EMBO J. 21, 1054-1062.

Brünger, A. T., Adams, P. D., Clore, G. M., DeLano, W. L., Gros, P., Grosse-Kunstleve, R. W., Jiang, J.-S., Kuszewski, J., Nilges, M., Pannu, M.S., Read, R. J., Rice, L. M., Simonson, T. \& Warren, G. L. (1998). Acta Cryst. D54, 905-921.

Chen, L., Chen, Li., Zhou, X. E., Wang, Y., Kahsai, M. A., Clark, A. T., Edmondson, S. P., Liu, Z., Rose, J. P., Wang, B.-C., Meehan, E. J. \& Shriver, J.W. (2004). J.Mol. Biol. 341, 73-91.

Dauter, Z., Dauter, M., de La Fortelle, E., Bricogne, G. \& Sheldrick, G. M. (1999). J. Mol. Biol. 289, 83-92.

Dauter, Z., Dauter, M. \& Dodson, E. (2002). Acta Cryst. D58, 494-506.

Debreczeni, J. É., Bunkóczi, G., Ma, Q., Blaser, H. \& Sheldrick, G. M. (2003). Acta Cryst. D59, 688696.

Debreczeni, J. É., Bunkóczi, G., Girmann, B. \& Sheldrick, G. M. (2003). Acta Cryst. D59, 393-395. 
de Graaff, R. A. G., Hilge, M., van der Plas, F. L. \& Abrahams, J. P. (2001). Acta Cryst. D57, 18571862.

Fu, Z.-Q., Rose, J. P. \& Wang, B.-C. (2004). Acta Cryst. D60, 499-506.

Fujinaga, M. \& Read, R. J. (1987). J. Appl. Cryst. 20, 517-521.

Gordon, E. J., Leonard, G. A., McSweeney, S. \& Zagalsky, P. F. (2001). Acta Cryst. D57, 1230-1237.

Hendrickson, W.A. \& Teeter, M. M. (1981). Nature (London), 290, 107-113.

Kiefersauer, R., Stetefeld, J., Gomis-Rüth, F. X., Romão, M. J., Lottspeich, F. \& Huber, R. (1996) J. Appl. Cryst. 29, 311-317.

Kiefersauer, R., Than, M. E., Dobbek, H., Gremer, L., Melero, M., Strobl. S,. Dias, J. M., Soulimane, T. \& Huber, R. (2000). J. Appl. Cryst. 33, 1223-1230.

Lartigue, A., Gruez, A., Briand, L., Blon, F., Bézirard, V., Walsh, M., Pernollet, J-C., Tegoni, M. \& Cambillau, C. (2004). J. Biol. Chem. 279, 4459-4464.

Lemke, C. T., Smith, G. D. \& Howell, P. L. (2002). Acta Cryst. D58, 2096-2101.

Li, S., Finley, J., Liu, Z.-J., Qiu, S.-H., Chen, H., Luan, C.-H., Carson, M., Tsao, J., Johnson, D., Lin, G., Zhao, J., Thomas, W., Nagy, L. A., Sha, B., DeLucas, L. J., Wang, B.-C. \& Luo, M. (2002). J. Biol. Chem. 277, 48596-48601.

Liu, Z.-J., Vysotski, E. S., Chen, C.-J., Rose, J. P., Lee, J. \& Wang, B.-C. (2000). Protein Sci. 9, $2085-$ 2093.

McRee, D. E. (1992). J. Mol. Graph. 10, 44-46.

Micossi, E., Hunter, W. N. \& Leonard, A. G. (2002). Acta Cryst. D58, 21-28.

Otwinowski, Z. \& Minor, W. (1997). Methods Enzymol. 276, 307-326.

Ramagopal, U. A., Dauter, M. \& Dauter, Z. (2003). Acta Cryst. D59, 1020-1027.

Sekar, K., Rajakannan, V., Velmurugan, D., Tamane, T., Thirumurugan, R., Dauter, M. \& Dauter, Z. (2004). Acta Cryst. D60, 1586-1590.

Sheldrick, G. M. (2002). Z. Kristallogr. 217, 644-650.

Sheldrick, G. M. (2003). SHELXC, Göttingen University, Germany.

Sheldrick, G.M., Hauptman, H.A., Weeks, C.M., Miller, M. \& Usón, I. (2001). International Tables for Crystallography, Vol. F, edited by E. Arnold \& M. G. Rossmann, pp. 333-351. Dordrecht: Kluwer Academic Publishers.

Terwilliger, T. C. (2000). Acta Cryst. D56, 965-972.

Terwilliger, T. C. (2003). Acta Cryst. D59, 38-44.

Terwilliger, T. C. \& Berendzen, J. (1999). Acta Cryst. D55, 849-861.

Wang, B.-C. (1985). Methods Enzymol. 115, 90-112.

Yang, C. \& Pflugrath, J. W. (2001). Acta Cryst. D57, 1480-1490.

Yang, C., Pflugrath, J. W., Courville, D. A., Stence, C. N. \& Ferrara, J. D. (2003). Acta Cryst. D59, 1943-1957.

Yao, M,. Zhou, Y. \& Tanaka, I. manuscript in preparation. 